\title{
Aphemia Related to a Premotor Cortex Infarction
}

\author{
S. Rheims ${ }^{a}$ N. Nighoghossian ${ }^{\text {a,c }}$ M. Hermier ${ }^{\text {b,c }}$ M. Formaglio ${ }^{\text {a }}$ S. Cakmak ${ }^{\text {a }}$ L. Derex $^{a}$ \\ P. Trouillas ${ }^{a}$
}

${ }^{a}$ Cerebrovascular Disease Center, ${ }^{b}$ Department of Radiology and ${ }^{C}$ Creatis UMR 5515, CNRS, Lyon, France

Dear Sir,

Aphemia was first defined by Broca [1] in 1861 as a defect in the faculty of articulated language. Moreover, the anatomic bases of clinical findings are unclear; some authors reported lesions involving Broca's area $[1,2]$, whereas functional imaging or some clinicoanatomic studies showed damage within the lateral premotor cortex $[3,4]$. We report 1 case of aphemia associated with an initial mutism linked to a lateral premotor cortex infarction.

A 70-year-old right-handed woman with a medical history of hypertension showed a sudden loss of speech. She was admitted to our stroke unit $6 \mathrm{~h}$ later. She was alert and fully oriented. She was unable to produce any sound. Comprehension of spoken language was normal. Her reading comprehension and her writing capacities were normal. Although she was still mute, we did not find any sign suggesting an opercular dysfunction: she was able to make kisses, blow air and stick out her tongue. She did not show any sign of buccofacial apraxia. There was no sign of hemiparesis. Monitored blood pressure was 150/80 mm Hg. Cardiovascular examination, ECT, chest X-ray and routine biological screening were normal.

MRI was performed on a Philips 1.5tesla MRI unit $7 \mathrm{~h}$ after the symptom onset. The protocol of imaging involved $T_{1}$, $\mathrm{T}_{2}, \mathrm{~T}_{2}{ }^{*}$ gradient echo, diffusion-weighted imaging, fluid-attenuated inversion recovery and magnetic resonance angiography which were obtained using the following parameters: $\mathrm{T}_{2}{ }^{*}$-weighted gradient echo sequence by $\mathrm{TR}=600-800 \mathrm{~ms}, \mathrm{TE}=$ $30-50 \mathrm{~ms}$ and flip angle $=10-20^{\circ}$.

Diffusion-weighted imaging (fig. 1) showed a left premotor cortex linear infarction involving the precentral sulcus and sparing Broca's area according to MRI mapping [5].

Two days later, her speech was hypophonic, slow and effortful with pauses between or inside morphemes. She had a monotonous tone with abnormal prosody. Although some phonemes were distorted, she had no phonemic or semantic paraphasias. The grammatical organization of sentences was normal. She did not express any mood disorders such as indifference or catastrophic reactions. She gradually improved; 2 months later, a mild impairment of speech persisted.

Our case was consistent with recent descriptions of aphemia characterized by a disturbance in articulation without any other language dysfunction [2]. Indeed, we observed neither linguistic impairment nor any sign of apraxia of speech supported by phonemic errors spontaneously recognized and corrected by the patient $[3,6]$. Although initial mutism in aphemia has been reported previously [2, 7], anatomical correlations between stroke and mutism are scarce. The pathophysiological basis of our observation is quite similar to those of the isolated aphemia which may involve a disruption of the connections between Broca's area and the region of control of the pharyngolaryngeal and tongue muscles. More specifically, functional im-

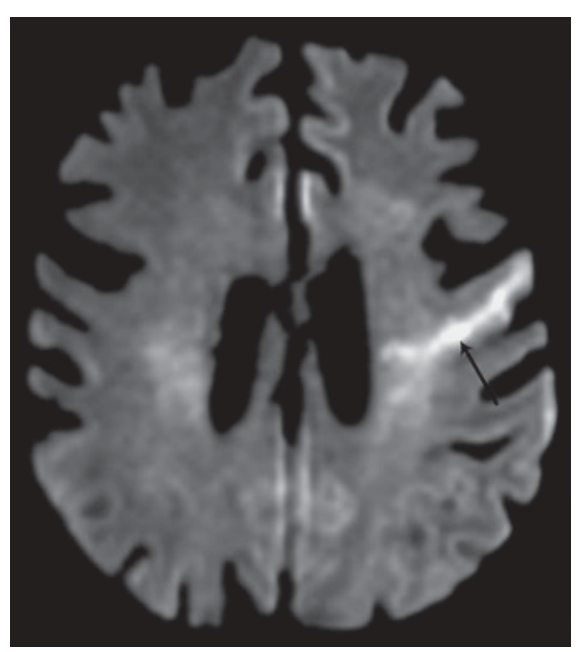

Fig. 1. Diffusion-weighted MRI showing a left premotor cortex infarction (arrow).

aging studies and clinicoanatomic studies $[3,4]$ have suggested that the left anterior insula and the lateral premotor cortex might serve as a preliminary processor to the initiation of an articulatory plan controlling the ability to shape the vocal tract for a particular speech sound. Thus, an acute lesion limited to the premotor cortex may destroy this ability to shape the vocal tract. However, in regard to the report of Hillis et al. [6], we cannot exclude a more extensive ischemic dysfunction of Broca's area.

\section{KARGER}

Fax +41613061234 E-Mail karger@karger.ch www.karger.com
(C) 2006 S. Karger AG, Basel 0014-3022/06/0554-0225\$23.50/0

Accessible online at: www.karger.com/ene
Prof. N. Nighoghossian

Hôpital Neurologique et Neurochirurgical Pierre-Wertheimer

59 , boulevard Pinel

FR-69003 Lyon (France)

Tel. +334723578 10, Fax +334723578 06, E-Mail norbert.nighoghossian@chu-lyon.fr 
References
1 Broca P: Remarques sur le siège de la faculté du langage articulé suivies d'une observation d'aphémie (perte de la parole). Bull Soc Anthropol 1865;2:330-357.

2 Schiff HB, Alexandre MP, Naeser MA, Galaburda AM: Aphemia: clinical-anatomic correlations. Arch Neurol 1983;40:720-727.

3 Dronkers NF: A new brain region for coordinating speech articulation. Nature 1996; 384:159-161.

4 Wise RJ, Greene J, Buchel C, Scott SK: Brain regions involved in articulation. Lancet 1999;353:1057-1061.
5 Talairach J, Tournoux P: Referentially Oriented Cerebral MRI Anatomy. New York, Thieme Medical Publishers, 1993.

6 Hillis AE, Work M., Barker PB, Jacobs MA, Breese EL, Maurer K: Re-examining the brain regions crucial for orchestrating speech articulation. Brain 2004;127:14791487.

7 Fox RJ, Kasner SE, Chatterjee A, Chalela JA: Aphemia: an isolated disorder of articulation. Clin Neurol Neurosurg 2001;103:123126. 